\title{
Impaired capsaicin-induced decrease in heart rate and coronary flow in isolated heart of diabetic rats
}

\section{J. Németh ${ }^{1}$, Z. Szilvássy ${ }^{2}$, G. Oroszi ${ }^{2}$, R. Pórszász ${ }^{2}$, B. Jakab¹, J. Szolcsányi ${ }^{1}$}

\author{
${ }^{1}$ Neuropharmacology Research Group of the Hungarian Academy of Sciences, \\ Department of Pharmacology and Pharmacoterapy, University of Pécs, Pécs, Hungary \\ ${ }^{2}$ Department of Pharmacology, University of Debrecen, Debrecen, Hungary
}

Received: February 15, 2002

Accepted: March 26, 2002

The effect of capsaicin $(0.1 \mu \mathrm{M})$ on heart rate and coronary flow was studied in Langendorff-perfused heart from streptozotocin-induced $(50 \mathrm{mg} / \mathrm{kg}$ i.v.) diabetic rats where sensory neuropathy developed.

In hearts from animals 4- and 8-week diabetes baseline heart rate and coronary flow decreased from $317.9 \pm 2.9$ b.p.m. and $13.4 \pm 0.7 \mathrm{ml} / \mathrm{min}$ to $255.1 \pm 12.7$ and $219.8 \pm 2.8$ b.p.m. and $8.9 \pm 0.6$ and $10.0 \pm 0.1 \mathrm{ml} / \mathrm{min}(P<0.05)$, respectively. Capsaicin significantly decreased both variables in either normal or 4-week diabetic animals its effects, however, on coronary flow or heart rate were missing in preparations from 8 -week diabetic rats. Endothelin-1 $(0.1 \mathrm{nM})$, the putative mediator of the capsaicin effect, significantly decreased heart rate and coronary flow irrespective of the presence or absence of diabetes. In the femoral nerve of streptozotocin-treated animals conduction velocity involving both fast conducting A- and slow-conducting C-fibres was decreased proportional to the duration of the pre-existing diabetic state.

It is concluded that in insulin deficient diabetes the diminished responses evoked by capsaicin on heart rate and coronary flow are signs of sensory neuropathy. This is related to a feeble endothelin release from sensory nerve endings without changes in post-receptor mechanisms mediating the endothelin effects.

Keywords: sensory nerve function, capsaicin, endothelin, diabetes mellitus, rat heart

Correspondence should be addressed to

Dr. János Szolcsányi

Department of Pharmacology and Pharmacoterapy

University of Pécs

H-7624 Pécs, Szigeti út 12, Hungary

Phone: +36 72536000 ext. 1601

Fax: +3672536218

E-mail: janos.szolcsanyi@aok.pte.hu 
A decrease in the release of sensory neuropeptides such as calcitonin gene-related peptide (CGRP), substance $\mathrm{P}$ and somatostatin in response to electrical stimuli is characteristic for diabetic neuropathy (11). Recently, this has been suggested to underlie a deficiency in several adaptive mechanisms attributed to the integrity of sensoryeffector function such as neurogenic inflammation $(8,12,27)$ and the ability of the heart to adapt to repetitive ischaemic insults i.e. the ischaemic preconditioning phenomenon $(5,24)$.

Capsaicin-stimulating VR-1 capsaicin (vanilloid) receptor $(1,19,20)$ located on a subpopulation of primary afferent fibres has been shown to decrease heart rate and coronary flow with an ensuing deterioration of cardiac function in isolated working rat hearts $(21,22)$. In isolated guinea-pig hearts, however, capsaicin increased the heart rate with coronary vasodilation and positive inotropy. As far as the neurotransmitters involved are concerned, nitric oxide and CGRP have been shown to mediate the capsaicin-induced positive cardiac effects in guinea-pigs $(6,13,14)$. In the rat heart the negative effects evoked by capsaicin are mediated by neural endothelin release $(21,22)$. Thus, we sought whether experimental diabetes associated with combined (sensory and motor) neuropathy induced by streptozotocin influenced the endothelin-mediated effects of capsaicin on isolated Langendorff-perfused rat heart.

\section{Materials and Methods}

\section{Ethics}

All experimental protocols applied in the present study conformed to the Europian Community guiding principles for the care and use of laboratory animals and were approved by the local ethical committee of Medical University of Pécs, Hungary.

\section{Experimental groups}

The experiments were carried out with 96 male Wistar rats weighing 300-350 g. The animals were housed in the Laboratory Animal Center of the Medical University of Pécs under pathogen free conditions provided with standard rat chow and tap water ad libitum. The animals were divided into four groups (Fig. 1). Groups 1 and 3: rats were treated with the solvent for streptozotocin 4 or 8 weeks before the experiments. The animals in Groups 2 and 4 were given streptozotocin $(50 \mathrm{mg} / \mathrm{kg}$ i.v.) at the same time. Each group consisted of 24 rats, with subgroups of 12 animals for studies on isolated heart preparations, 6 rats for plasma insulin and glucose levels, and a subset of 6 animals was used for nerve conduction velocity measurements. 
Determination of heart rate and coronary flow in Langendorff-perfused isolated rat hearts

It has been described in detail elsewhere (25). Briefly, rats were anaesthesized with diethyl ether. Na-heparin (500 IU/kg i.v.) was given to prevent blood coagulation. Following thoracotomy, the hearts were excised and mounted on a Langendorff apparatus. Following a $20 \mathrm{~min}$ period of aerobic perfusion with modified KrebsHenseleit solution, $0.1 \mu \mathrm{M}$ capsaicin or $0.1 \mathrm{nM}$ endothelin-1 was perfused for $5 \mathrm{~min}$. (Doses of drugs were selected according to the dose-response curves (22).) Coronary flow rate was measured by a timed collection of coronary effluent that dripped from the heart. Heart rate was also recorded by means of a Millar catheter inserted into the left ventricle via the left atrium and mitral valve. Baseline values were determined at the end of the equilibration period. The drug-induced changes were determined immediately after capsaicin/endothelin-1 perfusion. Both capsaicin and endothelin-1 exhibited a long-lasting effect, however, baseline values could be recaptured after a 2-hour washout period.

\section{Assessment of plasma insulin and glucose concentration}

Plasma insulin level was measured by radioimmunoassay (RIA) using commercial insulin RIA-kit. The glucose oxidase method was used for fasting blood glucose determination.

\section{Nerve conduction velocity}

We used the method described earlier (12). In brief, the left femoral nerve was prepared and stimulated with square-wave impulses $(500 \mu \mathrm{s}, 1-2 \mathrm{~Hz})$ applied through a pair of platinum electrodes. Another pair of electrodes was applied $2 \mathrm{~cm}$ distal to the stimulating electrodes for recording compound action potentials. The average time lags between stimulation signals and the appearance of corresponding " $\mathrm{A}$ " and " $\mathrm{C}$ " waves were determined. Dividing the distance between the stimulating and receiving electrodes by the interval between the stimulatory impulses (20 stimuli) and appearance of the corresponding "A" and "C" signals produced the data for evaluation $(9,12)$. 

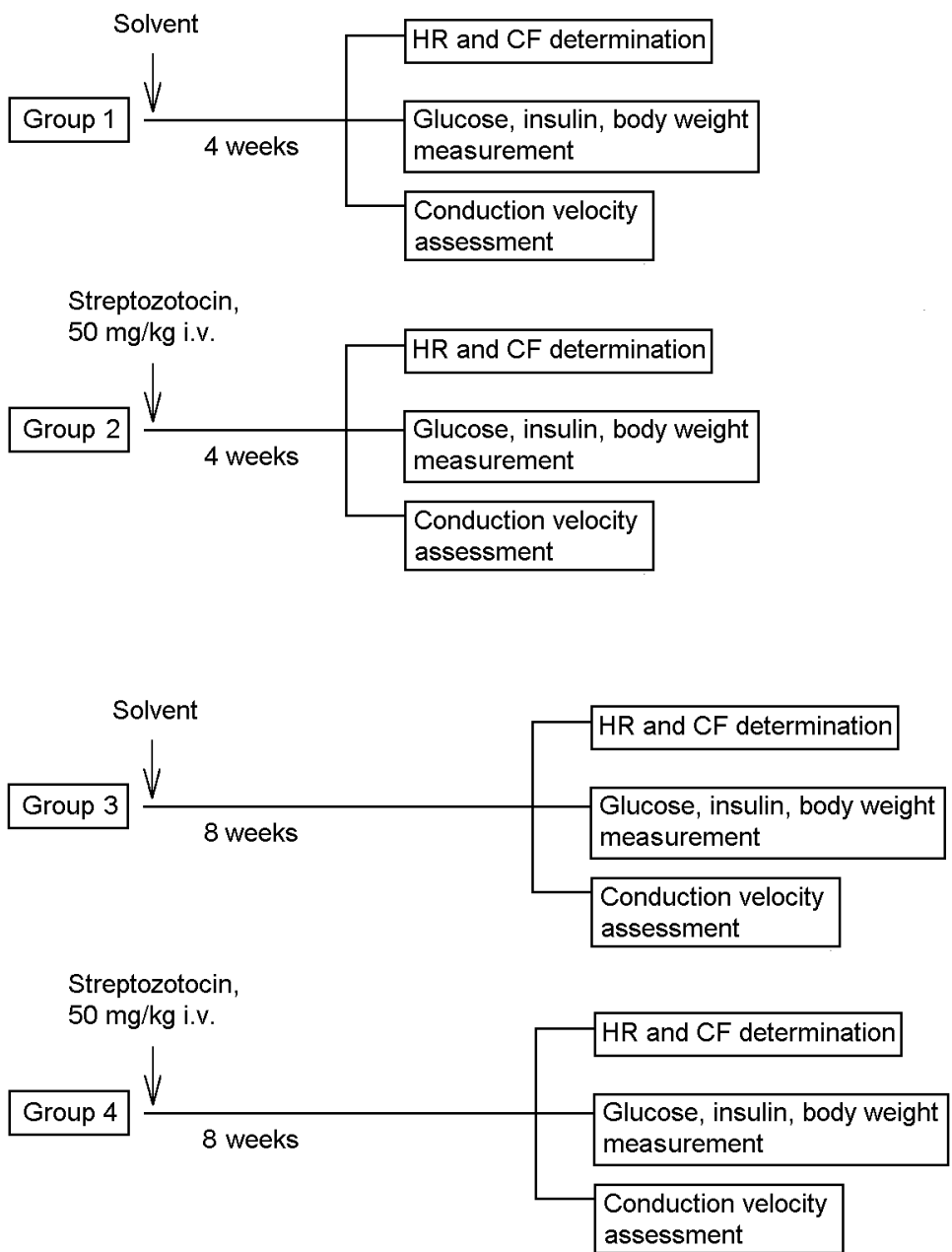

Fig. 1. Studies on the cardiac effects of capsaicin in rats with streptozotocin-induced diabetes. Study design. Abbreviations: HR: heart rate; CF: coronary flow (determinations in Langendorrf-preparations)

\section{Drugs and solutions}

Sodium thiopentone (Trapanal) was purchased from Byk Gulden (Konstanz, Germany), streptozotocin (Zanosar) from Upjohn (Kalamazoo, USA), diethyl ether and Tween 80 from Reanal (Budapest, Hungary), heparin Richter (Budapest, Hungary), capsaicin (8-methyl-N-vanillyl-6-nonenamide) and endothelin-1 from Sigma (St. Louis, USA), insulin RIA-kit (RK-400M) from Institute of Isotopes (Budapest, Hungary), all constituents of the modified Krebs-Henseleit solution from Merck (Darmstadt, 
Germany). Capsaicin was dissolved for a $1 \%$ (w/v) stock solution in a solution consisting of $10 \%$ (v/v) ethanol, $10 \%$ (v/v) Tween 80 and $80 \%(\mathrm{v} / \mathrm{v})$ saline. Further dilutions were made with saline.

\section{Statistical analysis}

The data are expressed as means \pm standard error of the mean (S.E.M.). Changes in heart rate and coronary flow were evaluated by means of analysis of variance (ANOVA) followed by a modified $t$-test for repeated measurements according to Bonferroni's method (26). Plasma insulin, glucose and sciatic nerve conduction velocity values were analyzed with Student's $t$-test for unpaired data. Changes were considered significant at $P<0.05$.

\section{Results}

Effects of diabetes on body weight, fasting blood glucose and plasma insulin levels

The animals in Groups 1 and 3 exhibited a continuous weight gain over the examination period, whereas the rats from Groups 2 and 4 revealed a moderate weight loss. Plasma insulin level significanly decreased with an ensuing increase in blood gucose level in the streptozotocin-treated groups at both 4 and 8 weeks after injection (Table I).

Nerve conduction velocity test

Nerve conduction velocity test was used as a "gold standard" of verifying diabetic neuropathy. Streptozotocin produced a decrease in conduction velocity in fast conducting myelinated A-fibres: $28.1 \pm 1.29 \mathrm{~m} / \mathrm{s}$ normal rats (Group 3) vs $15.3 \pm 1.66$ and $10.9 \pm 1.21 \mathrm{~m} / \mathrm{s}(P<0.05$ for both) in 4-week and 8-week diabetic animals (Groups 2 and $4)$, respectively. Slow conducting unmyelinated C-fibres disclosed a similar response.

Table I

Changes in body weight ( \pm denotes weight gain/loss), values of fasting blood glucose and plasma insulin levels in 4-week or 8-week diabetic rats and in the correspoding controls

\begin{tabular}{lrrrr}
\hline & Group 1 & Group 2 & Group 3 & Group 4 \\
\hline Changes in body weight $(\mathrm{g})$ & $+69.5 \pm 3.9$ & $-6.8 \pm 1.2$ & $+112.4 \pm 6.5$ & $-11.2 \pm 2.3$ \\
Fasting blood glucose $(\mathrm{mmol} / \mathrm{l})$ & $5.8 \pm 0.2$ & $18.1 \pm 2.2^{*}$ & $6.1 \pm 0.3$ & $21.1 \pm 3.1^{*}$ \\
Plasma insulin $(\mu \mathrm{IU} / \mathrm{ml})$ & $10.3 \pm 2.5$ & $2.6 \pm 0.5^{*}$ & $10.5 \pm 2.8$ & $2.7 \pm 0.8^{*}$ \\
\hline
\end{tabular}

Data are expressed as means \pm S.E.M.

$* P<0.05$, comparisons were made to the corresponding control groups. 
Conduction velocity was $0.64 \pm 0.09 \mathrm{~m} / \mathrm{s}$ in normal animals (Group 3 ) and it decreased to $0.44 \pm 0.03$ and $0.35 \pm 0.04 \mathrm{~m} / \mathrm{s}(P<0.05$ for both $)$ in 4 -week and 8 -week diabetic rats (Groups 2 and 4), respectively. The values of conduction velocity of fast and slow conducting fibres did not differ from each other in Groups 1 and 3.

Effect of diabetes on changes in heart rate and coronary flow in response to capsaicin or endothelin

Figure 2 shows that both the basal heart rate and coronary flow were significantly lower than those of the controls in hearts from animals treated with streptozotocin (Groups 2 and 4). Capsaicin $(0.1 \mu \mathrm{M})$ significantly decreased both variables in either normal (Group 3) or 4-week streptozotocin-diabetic (Group 2) animals. In preparations from 8-week diabetic (Group 4) rats, however, capsaicin failed to affect coronary flow or heart rate (Fig. 2A, B). Corresponding values obtained in hearts from the solvent treated groups ( 1 and 3 ) did not significantly differ from each other (data not shown).

Endothelin-1 $(0.1 \mathrm{nM})$ significantly decreased heart rate and coronary flow irrespective of the presence or absence of diabetes (Fig. 3A, B).

\section{Discussion}

These results are in accordance with previous findings that capsaicin decreases heart rate and coronary flow in isolated rat heart. The effects of capsaicin have been shown to result from a secondary sensory neurotransmitter release $(21,22)$. These data are in concert with the finding that the capsaicin (vanilloid) receptor subtype-1 (VR-1) the only vanilloid receptor described so far where capsaicin acts - is expressed on sensory neurons $(1,20)$. Regarding the mediator involved in the rat heart, the following evidence $(21,22)$ suggests a mediating role for endothelin: 1 . Capsaicin perfused at low concentrations through the isolated working rat heart released endothelin as detected from the coronary effluent. 2. Exogenous endothelin mimicked the cardiac and coronary effects of capsaicin. 3. The effects of capsaicin or endothelin were antagonized by the selective endothelin ETA receptor blocking agent BQ-123 and by the non-selective receptor antagonist PD 142893. 4. Removal of functional endothelial cells with Triton X-100 did not diminish the capsaicin-induced deleterious effects on cardiac function or on coronary flow. 5. Reduction of extracellular $\mathrm{Ca}^{++}$concentration prevented the release of endothelin and functional responses evoked by capsaicin, while the effects of endothelin remained unchanged. 6. The cardiac effects of capsaicin were 
absent in isolated heart preparations obtained from rats in which depletion of sensory neuropeptides was induced by systemic capsaicin treatment several days before the experiment.
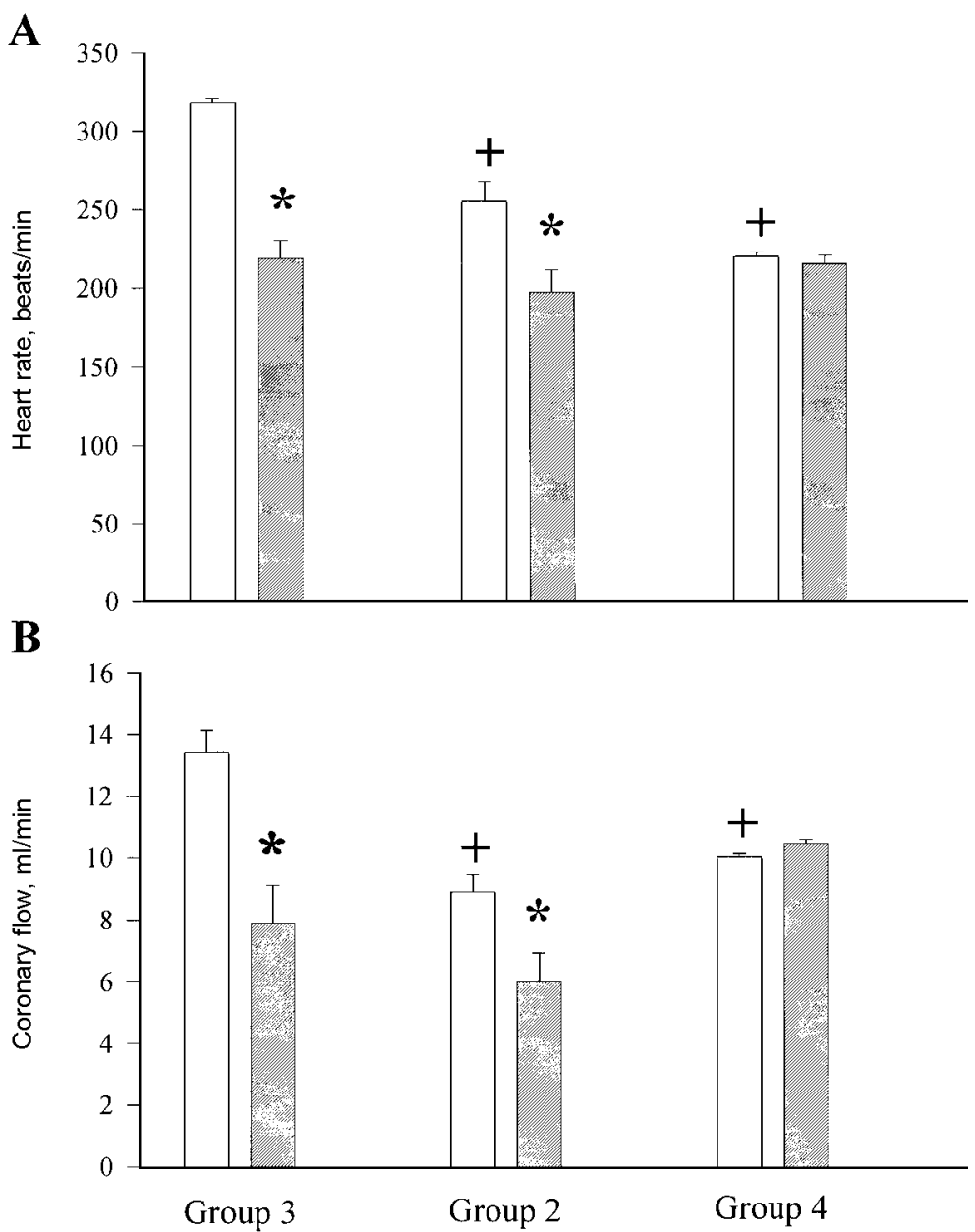

Fig. 2. Changes in heart rate (A) and coronary flow (B) in isolated Langendorff-perfused rat hearts in response to capsaicin. The first pair of columns represents data obtained from solvent treated animals (Group 3 ), the second pair of columns denotes values from 4-week diabetic rats (Group 2) and the third one designates data from 8-week diabetic animals (Group 4). Data are means \pm S.E.M., * indicates significant difference between baseline values (blank columns) and those following capsaicin exposure (hatched columns). + shows significant changes between baseline values of Group 3 compared to those of Group 2 and 4 (blank columns). $\mathrm{n}=6$ in each group. $* P<0.05,+P<0.05$ 


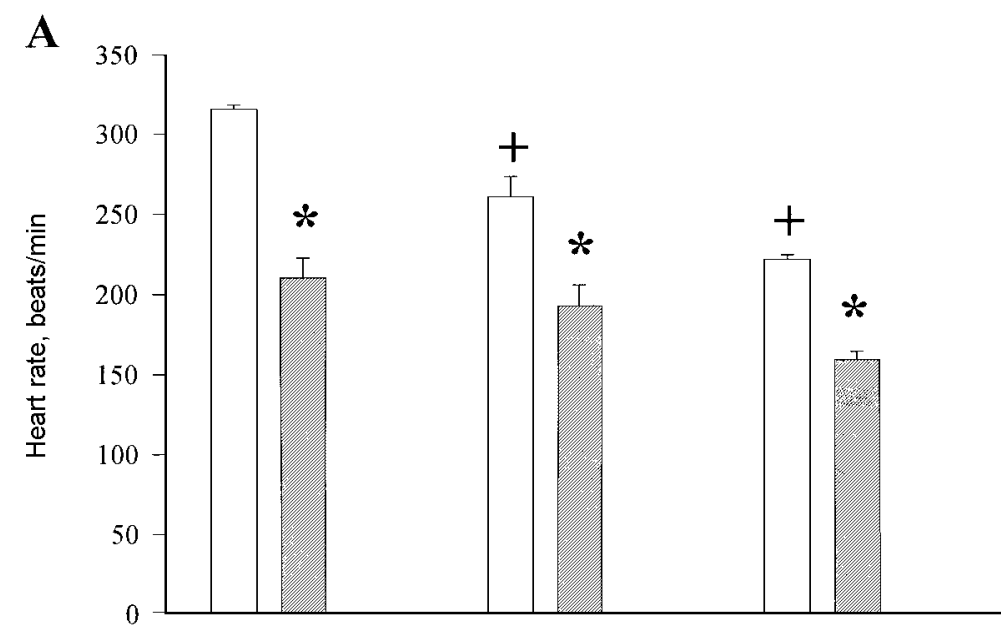

B

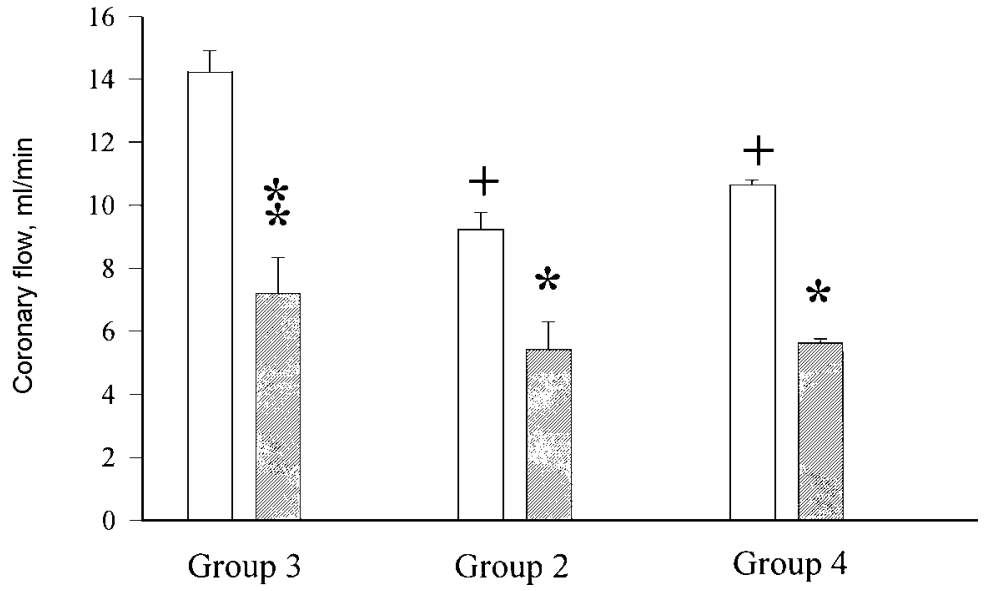

Fig. 3. Heart rate (A) and coronary flow (B) alterations by endothelin in Langendorff-perfused rat hearts. The first pair of columns shows data obtained with solvent treated animals (Group 3), the second pair of columns represents results from 4-week diabetic rats (Group 2) and the third-one denotes data from 8-week diabetic animals (Group 4). Data are means \pm S.E.M., * indicates significant difference between baseline values (blank columns) and those subsequent to endothelin exposure (hatched columns). + shows significant changes in baseline values of preparations from Group 3 compared to those from Groups 2 and 4 (blank columns). $\mathrm{n}=6$ in each group. ${ }^{*} P<0.05,+P<0.05$

According to the present experiments the cardiac effects of capsaicin are impaired with the development of diabetic neuropathy. Relatively preserved capsaicin effects were observed at an early diabetic state (4 weeks) but with the development of a significant degree of sensory neuropathy, significant attenuation of capsaicin responses occured in the heart of 8-week diabetic animals. 
Diabetes is known to produce several pathological changes in the cardiovascular system including changes in blood vessel structure, endothelial function, heart metabolism and neural dysfunction involving motor, sensory and autonomic nerves (15, $16,17)$. As far as the mechanism of sensory dysfunction in insulin-deficient diabetes is concerned, a defective axonal transport is thought to be the key initiating factor that eventually results in sensory nerve degeneration as a characteristic late complication of the disease (4). Diabetic neuropathy leads to a functional damage of sensory-effector machinery characterized by a significant decrease in sensory neurotransmitter release such as that of somatostatin, substance P and calcitonin gene-relat peptide (CGRP) that precedes neurodegenerative structural changes (11). Therefore, it is not surprising that important cardiovascular adaptive mechanisms such as ischaemic preconditioning known to be triggered by a release of sensory neurotransmitters in response to metabolic changes in the ischaemic myocardium such as CGRP and nitric oxide (NO) (5) are seriously impaired in diabetes (24).

Endothelin-like immunoreactivity of dorsal root ganglia is much higher than that of the stellate ganglion, spinal cord, heart, pulmonary artery or aorta. In fact it stored the highest level among the large range of tissues investigated (7). Endothelin is the principal sensory neurotransmitter which mediates the effects of capsaicin in isolated rat heart $(21,22)$. In hearts from diabetic rats the capsaicin-induced decrease in heart rate and coronary flow were attenuated while similar responses evoked by endothelin in diabetic animals did not differ from those of the controls. These data indicate that diabetes caused peripheral sensory neural dysfunction in the rat heart while postreceptor signalling pathways for endothelin remained unaltered. Endothelin may act both on smooth muscle cells of the coronary arteries and on endothelial receptors mediating opposite vascular effects i.e. vasoconstriction by stimulation smooth muscle receptors and vasodilation by releasing endothelial vasorelaxants (2). It is clearly shown in (Figs $2 \mathrm{~B}$ and $3 \mathrm{~B}$ ) that baseline coronary flow is significantly decreased in diabetic hearts, thus, it is concievable to suppose that coronary vasoconstriction by endothelin may even be exaggerated in diabetes. In our experiments, however, the decrease in coronary flow and heart rat were similar in hearts of diabetic rats as those of the controls. Irrespective of the degree of impaired endothelial fuction which might contribute to vasoconstrictive responses to either endothelin or capsaicin in the diabetic rat heart, the results show that function of both cardiac sensory nerves and coronary endothelium is significantly impaired in steptozotocin diabetes of 8-week duration in rats. Besides a significant attenuation of cardiac capsaicin effects and a decrease in baseline coronary flow, the streptozotocin-treated rats had characteristic features of Type I diabetes mellitus in that they failed to gain weight and exhibited very low plasma insulin levels associated with hyperglycaemia. Moreover, nerve conduction velocity studies revealed sensory neuropathy proportional to the duration of diabetes. 
The reduction of the capsaicin effects in diabetes are highly consistent with observations demonstrating a depletion of neurotransmitters (3) and a decreased neuropeptide release from sensory nerves in response to a standardized challenge in rats with streptozotocin diabetes (11). The rich sensory innervation of the heart raised the possibility that these nerves are fundamentally involved in development of the ischaemic preconditioning phenomenon $(5,23,28)$, the most powerful cardioprotective mechanism described to date (10). Epidemiological studies have clearly demonstrated that patient with either insulin-dependent diabetes mellitus or non-insulin-dependent diabetes mellitus are more prone to myocardial infarction and post-myocardial complications independ of whether or not they have coronary atherosclerosis (18). It has been known for a long time that diabetes is an independent risk factor for ischaemic heart disease, but there is much debate as to whether how the diabetic heart becomes more susceptable to ischaemic injury. A study in which the evolution of diabetes in relation to ischaemia-reperfusion injury was investigated, the diabetic rat heart was found more resistant to ischaemia-reperfusion-injury in the early phase of diabetes (2week), but this additional protection was then lost after an additional 4-6-week diabetic period. The animals which had been diabetic for eight weeks, a worse outcome from ischaemia-reperfusion occured as compared to hearts from normal animals (24). Our results closely correlate with these data, since responses to capsaicin were not significantly impaired in 4-week diabetic animals, however, the capsaicin-induced changes were completely abolished after a pre-existing 8-week diabetic period. Taken together the role of sensory nerves in the adaptability of the heart to ischaemia and the impairment of these nerves in diabetes with the loss of the preconditioning phenomenon in advanced diabetic state, it seems suggestive that functional impairment of sensory nerves underlies the decrease in the capability of the heart to adapt to repetitive ischaemic insults in an advanced diabetic state.

\section{Acknowledgements}

The present work was supported by the Hungarian Academy of Sciences and Hungarian Research Grants: OTKA T-029428, T-032002; NRDP 1/047/2001. The authors are grateful to Mrs Csilla Zádor for the expert technical assistance.

\section{REFERENCES}

1. Caterina MJ, Schumacher MA, Tominaga M, Rosen TA, Levine JD, Julius D: The capsaicin receptor: a heat-activated ion channel in the pain pathway. Nature 389, 816-824 (1997)

2. Chokkukannan J, Zeitlin IJ, Wainwright CL: Modulation of endothlin-1 release by a transmissible factor from ischemic myocardium. J. Cardiovasc. Pharmacol. 31, S427-S430 (1998) 
3. Diemel LT, Stevenc EJ, Willars GB, Tomlinson DR: Depletion of substance P and calcitonine generelated peptide in sciatic nerve of rats with experimental diabetes; effects of insulin and aldose reductase inhibition. Neurosci. Lett. 137, 253-256 (1992)

4. Fedele D, Giugliano D: Peripheral diabetic neuropathy: current recommendations and future prospects for its prevention and management. Drugs 54, 414-421 (1997)

5. Ferdinandy P, Csont T, Csonka C, Torok L, Dux M, Nemeth J, Horvath JI, Szilvassy Z: Capsaicinsensitive local sensory innervation is involved in pacing-induced preconditioning in rat hearts: role of nitric oxide and CGRP? Naunyn-Schmiedeberg's Arch. Pharmacol. 356, 356-363 (1997)

6. Franco-Cereceda A: Calcitonine gene-related peptide and tachykinins in relation to local sensory control of cardiac contractility and coronary vascular tone. Acta Physiol. Scand. Suppl. 569, 1-63 (1988)

7. Franco-Cereceda A, Rydh M, Lou YP, Dalsgaard CJ, Lundberg JM: Endothelin as a putative sensory neuropeptide in the guinea-pig: different properties in comparison with calcitonin gene-related peptide. Regul. Peptides 32, 253-265 (1991)

8. Gyorfi A, Fazekas A, Feher E, Ender F, Rosivall L: Effects of streptozotocin-diabetes on neurogenic inflammation of gingivomucosal tissue in rat. J. Periodontal Res. 31, 249-255 (1996)

9. Janig W, Lisney SJW: Small diameter myelinated afferents produce vasodilatation but not plasma extravasation in rat skin. J. Physiol. 415, 477-486 (1989)

10. Lawson CS, Downey JM: Preconditioning: state of the art myocardila protection. Cardiovasc. Res. 27, 542-550 (1993)

11. Németh J, Szilvássy Z, Than M, Oroszi G, Sári R, Szolcsányi J: Decreased sensory neuropeptide release from trachea of rats with streptozotocin-induced diabetes. Eur. J. Pharmacol. 369, 221-224 (1999a)

12. Németh J, Than M, Sári R, Peitl B, Oroszi G, Farkas B, Szolcsányi J, Szilvássy Z: Impairment of neurogenic inflammatory and anti-inflammatory responses in diabetic rats. Eur. J. Pharmacol. 386, 83-88 (1999b)

13. Oroszi G, Szilvássy Z, Németh J, Ferdinándy P, Szolcsányi J, Tosaki A: Interaction between capsaicin and nitrate tolerance in isolated guinea-pig heart. Eur. J. Pharmacol. 368, R1-R3 (1999a)

14. Oroszi G, Szilvássy Z, Németh J, Tosaki A, Szolcsányi J: Interplay between nitric-oxide and CGRP by capsaicin in isolated guinea-pig heart. Pharmacol. Res. 40, 125-128 (1999b)

15. Paulson DJ: The diabetic heart is more sensitive to ischemic injuri. Cardiovasc. Res. 34, 104-112 (1997)

16. Scheen AJ: Drug treatment of non-insulin-dependent diabetes mellitus in the 1990s: achievements and future developments. Drugs 54, 355-368 (1997)

17. Stehouwer CDA, Lambert J, Donker AJM, van Hinsbergh VWM: Endothelial dysfunction and pathogenesis of diabetic angiopathy. Cardiovasc. Res. 34, 55-68 (1997)

18. Stone PH, Müller JE, Hartwell T, York BJ, Rutherford JD, Parker CB, Turi ZG, Strauss HW, Willerson JT, Robertson T: The effect of diabetes mellitus on prognosis and serial left ventricular function after acute myocardial infarction: contribution of both coronary disease and diastolic left ventricular dysfunction to the adverse prognosis. The MILIS Study Group. J. Am. Coll. Cardiol. 14, 49-57 (1989)

19. Szállási Á, Blumberg PM: Vanilloid (capsaicin) receptors and mechanisms. Pharmacol. Rev. 51, 159-211 (1999)

20. Szolcsányi J (2002): Future perspectives of capsaicin research. In: Medicinal and Aromatic Plants, Vol. Capsicum, ed. Amit K, Harwood Academic Publications (in press)

21. Szolcsányi J, Oroszi G, Németh J, Szilvássy Z, Blasig IE, Tosaki A: Functional and biochemical evidence for capsaicin-induced neural endothelin release in isolated working rat heart. Eur. J. Pharmacol. 419, 215-221 (2001)

22. Szolcsányi J, Oroszi G, Németh J, Szilvássy Z, Tosaki A: Endothelin release by capsaicin in isolated working rat heart. Eur. J. Pharmacol. 376, 247-250 (1999) 
23. Tang ZL, Dai W, Li YJ, Deng HW: Involvement of capsaicin-sensitive sensory nerves in early and delayed cardioprotection induced by brief ischaemia of the small intestine. Naunyn-Schmiedeberg's Arch. Pharmacol. 359, 243-247 (1999)

24. Tosaki A, Engelman DT, Engelman RM, Das DK: The evolution of diabetic response to ischemia/reperfusion and preconditioning in isolated working rat hearts. Cradiovasc. Res. 31, 526-536 (1996)

25. Tosaki A, Szerdahelyi P, Engelman RM, Das DK: Potassium channel openers and blockers: do they possess proarrhythmic or antiarrhythmic activity in ischemic and reperfused heart? J. Pharmacol. Exp. Ther. 267, 1355-1362 (1993)

26. Wallenstein S, Zucker CL, Fleiss JL: Some statistical methods useful in circulation research. Circ. Res. 47, 1-9 (1980)

27. Walmsley D, Wiles PG: Early loss of neurogenic inflammation in the human diabetic foot. Clin. Sci. Colch. 80, 605-610 (1991)

28. Zhou FW, Li YJ, Lu R, Deng HW: Protection of calcitonine gene-related peptide-mediated preconditioning against coronary endothelial dysfunction induced by reperfusion in the isolated rat heart. Life Sci. 64, 1091-1097 (1999) 